\title{
Situación del cuidador y características del cuidado de personas con discapacidad en Santiago de Cali
}

\author{
YANETH FIGUEROA ${ }^{(1)}$
}

\section{RESUMEN}

Introducción. El cuidado es una actividad inherente al ser humano, el cuidado informal es aquel que es realizado por un familiar o por personas cercanas que no reciben remuneración $y$, en general, no es reconocido. Material y Método. El presente estudio describe las características sociodemográficas del cuidador y de la persona que recibe el cuidado, la situación en torno al cuidado, explora sus necesidades y demandas de apoyo del cuidador, así como la descripción de las rutinas que involucran el cuidado. Con este propósito, se llevó a cabo un estudio de tipo descriptivoexploratorio, con visitas a 83 familias seleccionadas a través de un muestreo no probabilístico a partir de una base de datos inicial y, posteriormente, fueron encuestados 36 cuidadores que pertenecían a la comuna 18 de la ciudad de Santiago de Cali. Resultados. Los resultados evidencian una población de predominio femenino, baja escolaridad, edad adulta tanto en cuidadores y receptores de cuidado, con vínculo familiar. Conclusión. La mayor proporción de cuidadores se ocupa de las tareas del hogar (80\%), reporta antecedentes patológicos (50\%), no recibe remuneración económica por el cuidado el (82\%), y manifiesta que requiere algún tipo de apoyo (61\%).

Palabras clave: Discapacidad, cuidador, cuidado informal, necesidades, apoyos.

\section{ABSTRACT}

\section{CAREGIVER SITUATION AND CHARACTERISTICS OF CAREGIVING OF DISABLED PEOPLE IN SANTIAGO DE CALI}

Introduction: Caregiving is an inherent human activity, and informal caregiving is unpaid, often unrecognized, care given by the family and friends of the ill or disabled person. Materials and Methods: This study describes the sociodemographic characteristics of caregivers as well as the person receiving the care, and the caregiving situation and its context. It explores caregivers' needs for support as well as their caregiving rutines. With this goal in mind, a descriptive-exploratory study was carried out, starting with visits to 83 families selected through non-random sampling of an initial database. Later, 36 caregivers from county 18 of Santiago de Cali were chosen and surveyed. Results: The results show a predominantly female population, with low levels of education, adult age for both caregivers and care receivers, and a family link between caregiver and care receiver. Conclusion: The majority of caregivers also are responsible for other domestic tasks (80\%), report a history of disease (50\%), do not receive payment for caregiving (82\%), and require some kind of support (61\%).

Key words: Disability, caregiver, informal caregiving, needs, support

(1) Universidad Santiago de Cali. Kra 67 No3C-15 apto 403. Unidad Residencial Ariguami Barrio El Refugio.

yaneth.figueroa@gmail.com 


\section{INTRODUCCIÓN}

El marco situacional de las personas que participan en la toma de decisiones, apoyan y cuidan $^{1}$ a quienes se encuentran en situación de discapacidad, plantea aspectos inherentes a las necesidades y demandas de este grupo, donde se espera que las respuestas a estas necesidades vayan de acuerdo con las condiciones y características del entorno familiar y comunitario. Este marco define un contexto en la relación del cuidador y de quien recibe el cuidado, configurando un escenario que advierte múltiples condiciones de vulnerabilidad y de invisibilidad ${ }^{2,3}$, con necesidades y demandas que convocan a la reivindicación en la vía de sus derechos y libertades individuales fundamentales en el logro de un desarrollo armónico, digno, personal y social de este grupo de personas, para lo cual es necesario que se convierta en objeto de políticas públicas ${ }^{4}$.

La actividad de cuidado se encuentra en estrecha relación con una serie de factores que incluyen la situación de discapacidad, la transición demográfica, las nuevas reformas de salud y el desplazamiento del cuidado formal hacia el informal, el papel de cuidador, su relación con el género y las inequidades en salud, entre otras ${ }^{5}$.

Con respecto a la transición epidemiológica y demográfica, se calcula que para 2050 el total de la población sea cercano a los 72 millones, la esperanza de vida sea ligeramente superior a los 79 años y los mayores de 60 años superen el $20 \%{ }^{6}$; grupo poblacional que registra epidemiológicamente la mayor carga de enfermedad a expensas de las enfermedades crónicas no transmisibles (ECNT) ${ }^{7}$.

En Colombia, las ECNT representan la primera causa de muerte después de los 45 años en el $72 \%$ de los departamentos. En el Municipio de Cali, siete de las diez primeras causas de mortalidad se debieron a causas crónicas como enfermedades cardiovasculares, hipertensión arterial, diabetes y cáncer de estomago, entre otros $^{8}$. Sumado a esto, los datos nacionales de prevalencia de discapacidad en un $6,4 \%{ }^{9}$, estas situaciones plantean una condición de doble discapacidad, la cual irá en aumento conllevando a una mayor dependencia y, por lo tanto, a una mayor necesidad de cuidado.

De otro lado, los cambios en el sistema de seguridad social y en la provisión de servicios por parte del Estado, han generado la pérdida del rol protagónico en la provisión del cuidado, transfiriéndolo a la esfera individual y familiar, constituyéndola en pilar fundamental del cuidado, ${ }^{10}$ hasta constituirse en una situación informal, presentando rasgos característicos que afectan su visibilidad y reconocimiento social ${ }^{11}$.

Por tanto, las políticas y planes gubernamentales deben formular estrategias encaminadas hacia el bienestar, favoreciendo las potencialidades de las familias y las de las personas con discapacidades. En este sentido, la Política Nacional de Discapacidad y las políticas públicas municipales proponen mejorar el estado salud, disminuir riesgos y disminuir inequidades en salud ${ }^{12}$. Por consiguiente, se hace necesario conocer los escenarios donde se desarrollan actividades de cuidado realizadas por las familias, con el objetivo de guiar e implementar las estrategias para el mejoramiento de la salud ${ }^{13}$.

\section{MATERIAL Y MÉTODO}

Se realizo un estudio descriptivo de corte exploratorio, que permitió reconocer las características sociodemográficas del cuidador y de los receptores de cuidado, condición de salud auto referida por el cuidador, elementos del cuidado y las actividades en torno al cuidado, las formas de apoyo y necesidades.

La población estuvo constituida por familias que refirieron tener en su hogar personas en situación de discapacidad, captadas por medio de la base de datos inicial, del estudio desarrollado en la comuna 18 de Cali por el grupo de salud pública de la Universidad San Martín durante 2008, sobre factores de riesgo y macro determinantes de la salud. La muestra fue calculada mediante un muestreo no probabilístico en función de la base de datos, con 
un porcentaje de error 5\%, nivel de confianza $95 \%$, proporción esperada $50 \%$. Fueron visitadas 83 familias en sus hogares, en 48 de ellas, no existía persona con discapacidad o el tipo de discapacidad no requirió cuidador. Finalmente fueron encuestados 36 cuidadores.

Para la recolección de los datos se diseñó un cuestionario inicial, al cual se le realizó un análisis del contenido y pruebas de consistencia interna. Se incluyeron como variables: características sociodemográficas, estado de salud del cuidador y receptor del cuidado, con base en la encuesta de calidad de vida de $2003^{14}$ y el cuestionario de salud de Madrid 2007 ${ }^{15}$; elementos propios del cuidado, necesidades percibidas por el cuidador y apoyo familiar, con base en el cuestionario sobre Red Social de Terán-Trillo $2007^{16}$. Se realizó prueba piloto que permitió reconocer barreras de lenguaje, exhaustividad de categorías presentes en el instrumento, emergencia de variables y finalmente reconocimiento de algunos elementos del contexto que fueron atendidos.

Para la entrada de los datos se uso el programa Epi Info versión 6.0, realizando una doble digitación con el fin de evitar los posibles errores. Se chequearon errores mediante la impresión de las tablas de datos, buscando inconsistencias. En los casos necesarios se verificó la información con nuevas visitas y llamadas telefónicas. Se realizó un análisis univariado $\mathrm{y}$, posteriormente, una exploración asociativa utilizando el estadístico de Fischer, de acuerdo con los resultados relevantes.

\section{RESULTADOS}

Tanto en el cuidador como en la persona receptora predominó el género femenino, edad avanzada y el bajo nivel de escolaridad. (Tablas 1 y 3). La discapacidad motora y múltiple fueron los principales tipos y la enfermedad general la primera causa (Tabla 2).

El 58.3\% de los cuidadores se dedica exclusivamente a las actividades del hogar; el 11.1\% a hogar y trabajo, independiente, el $11.2 \%$ además del hogar se ayuda para el sostenimiento con ventas en casa, sólo el $11.1 \%$ es empleada.
Tabla 1. Características sociodemográficas de las personas receptoras de cuidado.

\begin{tabular}{|c|c|c|c|}
\hline \multicolumn{2}{|c|}{ Variables } & \multirow{2}{*}{$\begin{array}{l}\begin{array}{l}\text { Fre- } \\
\text { cuencia }\end{array} \\
25\end{array}$} & \multirow{2}{*}{$\begin{array}{l}\begin{array}{l}\text { Por- } \\
\text { centaje }\end{array} \\
69,4 \%\end{array}$} \\
\hline & Femenino & & \\
\hline & Masculino & 11 & $30,6 \%$ \\
\hline \multirow{5}{*}{ Edad } & $\begin{array}{l}\text { Menores de } 18 \\
\text { años }\end{array}$ & 5 & $13,9 \%$ \\
\hline & $\begin{array}{l}\text { Entre } 18 \text { y } 25 \\
\text { años }\end{array}$ & 1 & $2,8 \%$ \\
\hline & $\begin{array}{l}\text { Entre } 26 \text { y } 45 \\
\text { años }\end{array}$ & 6 & $16,7 \%$ \\
\hline & $\begin{array}{l}\text { Entre } 46 \text { y } 65 \\
\text { años }\end{array}$ & 7 & $19,4 \%$ \\
\hline & $\begin{array}{l}\text { Mayores de } 65 \\
\text { años }\end{array}$ & 17 & $47,2 \%$ \\
\hline \multirow{5}{*}{ Estado civil } & Soltero & 18 & $50,0 \%$ \\
\hline & Casado & 6 & $16,7 \%$ \\
\hline & Unión libre & 1 & $2,8 \%$ \\
\hline & separado & 1 & $2,8 \%$ \\
\hline & Viudo & 10 & $27,8 \%$ \\
\hline \multirow{4}{*}{$\begin{array}{l}\text { Nivel de } \\
\text { estudios }\end{array}$} & Primaria & 22 & $61,1 \%$ \\
\hline & Secundaria & 1 & $2,8 \%$ \\
\hline & Ninguna & 9 & $25,0 \%$ \\
\hline & No sabe & 4 & $11,1 \%$ \\
\hline Total & & 36 & $100 \%$ \\
\hline
\end{tabular}

Esto indica que $80 \%$ de los cuidadores está presente en el hogar y que el $86.1 \%$ no recibe remuneración económica por el hecho de cuidar.

Con respecto al tiempo dedicado a las actividades de cuidado, los cuidadores en su mayoría cumplen su labor por seis o más años como se puede apreciar en la Tabla 4. 
Tabla 2. Características de la discapacidad de la persona receptora de cuidado.

\begin{tabular}{|l|l|c|c|}
\hline \multicolumn{1}{|l|}{ Variables } & Frecuencia & Porcentaje \\
\hline \multirow{4}{*}{$\begin{array}{l}\text { Causa de la } \\
\text { discapacidad }\end{array}$} & Complicaciones de parto & 6 & $16,7 \%$ \\
\cline { 2 - 4 } & Enfermedad general & 18 & $50,0 \%$ \\
\cline { 2 - 4 } & Alteración genética & 3 & $8,3 \%$ \\
\cline { 2 - 4 } & Accidente & 4 & $11,1 \%$ \\
\cline { 2 - 4 } & No sabe & 5 & $13,9 \%$ \\
\hline \multirow{5}{*}{ Tipo de discapacidad } & Motora & 11 & $30,6 \%$ \\
\cline { 2 - 4 } & Sensorial & 4 & $11,2 \%$ \\
\cline { 2 - 4 } & Cognitiva & 4 & $11,2 \%$ \\
\cline { 2 - 4 } & Múltiple & 16 & $44,4 \%$ \\
\cline { 2 - 4 } & Mental & 1 & $2,8 \%$ \\
\hline
\end{tabular}

Tabla 3. Características sociodemográficas de los cuidadores.

\begin{tabular}{|c|c|c|c|}
\hline \multicolumn{2}{|l|}{ Variables } & \multirow{2}{*}{$\begin{array}{c}\text { Frecuencia } \\
31\end{array}$} & \multirow{2}{*}{$\begin{array}{c}\text { Porcentaje } \\
86,1 \%\end{array}$} \\
\hline & Mujer & & \\
\hline Genero & Hombre & 5 & $13,9 \%$ \\
\hline \multirow{2}{*}{ Estrato socioeconómico } & Uno y Dos & 24 & $66.6 \%$ \\
\hline & Tres & 12 & $33,3 \%$ \\
\hline \multirow{4}{*}{ Edad } & Entre 26 y 35 años & 8 & $22,2 \%$ \\
\hline & Entre 36 y 45 años & 4 & $11,1 \%$ \\
\hline & Entre 46 y 65 años & 18 & $50,0 \%$ \\
\hline & Mayores de 65 años & 6 & $16,7 \%$ \\
\hline \multirow{5}{*}{ Estado civil } & Soltero & 10 & $27,8 \%$ \\
\hline & Casado & 16 & $44,4 \%$ \\
\hline & Unión libre & 6 & $16,7 \%$ \\
\hline & Separado & 1 & $2,8 \%$ \\
\hline & Viudo & 3 & $8,3 \%$ \\
\hline \multirow{4}{*}{ Nivel de estudios } & Primaria & 18 & $50,0 \%$ \\
\hline & Secundaria & 14 & $38,9 \%$ \\
\hline & Técnicos & 3 & $8,3 \%$ \\
\hline & Ninguno & 1 & $2,8 \%$ \\
\hline Total & & 36 & $100 \%$ \\
\hline
\end{tabular}


Tabla 4. Tiempo que lleva realizando la actividad de cuidado.

\begin{tabular}{|l|c|c|}
\hline Variable & frecuencia & Porcentaje \\
\hline Tiempo de cuidado & & \\
\hline Menos de un año & 4 & $11,1 \%$ \\
\hline De dos a cinco años & 10 & $27,8 \%$ \\
\hline De seis a diez años & 8 & $22,2 \%$ \\
\hline Más de diez años & 14 & $38,9 \%$ \\
\hline Total & 36 & $100,0 \%$ \\
\hline
\end{tabular}

En relación al estado de salud, el $80.6 \%$ de los cuidadores manifestó sentirse saludable, sin embargo el $55.6 \%$ expresó que ha consultado al médico en los últimos 6 meses. El $61.1 \%$ de los cuidadores reportó diagnóstico de alguna patología, enfermedades crónicas no trasmisibles en un $38.9 \%$. El $50 \%$ de a quienes se les ha diagnosticado alguna patología no recibe tratamiento médico.

Los cuidadores realizan más de una actividad de la vida diaria para ayudar a la persona a su cuidado, el $30.65 \%$ desempeña todas las actividades (aseo, alimentación, traslados dentro y fuera del hogar, administración de medicamentos).

El 80\% de los cuidadores reconoce que ha dejado de lado alguna actividad por el hecho de cuidar, tal como trabajar y estudiar. El 78\% no conoce ninguna institución o programa de apoyo, el $61 \%$ no sabe dónde acudir para solicitar ayuda, y el $25 \%$ no tiene con quién dejar a la persona que cuida.

Entre las necesidades reconocidas por el cuidador, el $25 \%$ requiere apoyo económico, el $11.1 \%$ asesorías y capacitación, el $25 \%$ manifiesta necesitar de todas (salud, trabajo, económico, asesorías y capacitación).

Por otro lado, el 13,9\% reconoce que recibe apoyo de tipo material, el 11.1\% compañía exclusivamente. El 19.4\% recibe compañía y afecto y el $16.7 \%$ servicios y otros (llevar o traer cosas). El $16.7 \%$ recibe apoyo de hijos exclusivamente, el 13.7\% (hijos, madre, cónyuge), $28 \%$ otros familiares, cónyuge $8.3 \%$, hermanos $8,3 \%$, discapacitado $5.6 \%$.

Se encontró diferencias significativas $(p=0,020)$ entre sensación de salud del cuidador y antecedentes de enfermedades. Así como entre la sensación de fatiga y dolores musculares, $(p=0,016)$, contrario al hecho de tener otras personas a cargo o haber consultado al médico, donde no se encontraron diferencias estadísticas significativas.

De igual forma se encontró relación entre menor sensación de fatiga manifestada y haber recibido ayuda. $(\mathrm{p}=0.008)$.

\section{DISCUSIÓN}

Este estudio demostró que los cuidadores en su mayoría son mujeres (86\%), amas de casa $(58.3 \%)$ y con estudios primarios $(50 \%)$. Hecho reportado en otros estudios donde se plantea que el cuidado de la salud de la comunidad es patrimonio casi exclusivo de las mujeres, lo que supone un elemento de desigualdad de género en salud ${ }^{5,11}$. Esta desigualdad de género en el campo del cuidado, aumenta las dificultades para que sea considerado un aspecto importante, considerando la poca representación institucional en los espacios públicos del género femenino.

Entre los hallazgos importantes de este estudio se destaca el alto porcentaje de cuidadores que ha dejado de realizar actividades por el hecho de cuidar (trabajo, estudio) y no reciben remuneración económica por la actividad de cuidado. Estas condiciones del cuidado, sumado al tiempo dedicado a esta actividad por años, a la responsabilidad por otras personas y por el cuidado del hogar, dan indicios de la alta exigencia que representa el cuidado para quienes lo ejercen. Estos aspectos han sido objeto de otros estudios, reconociendo la importancia de los costos que implica el cuidado y la contribución de los cuidados familiares como recursos dentro de la macroeconomía y la necesidad de cuidarlos ${ }^{10}$.

En cuanto a la salud, la mayoría de los cuidadores refiere sentirse saludable. Sin embargo, resulta contradictorio que más de la mitad de ellos reporte que ha consultado al médico y 
que -en su mayoría- refiere algún tipo de enfermedad y la presencia de dolor en alguna parte de su cuerpo. En este sentido, algunos estudios destacan la influencia que tiene el hecho de cuidar sobre la salud del cuidador y por consiguiente en la calidad de vida ${ }^{19,20}$. Influencia que se pudo determinar en la relación de las variables planteadas, donde el presentar antecedentes de enfermedades, influye sobre la sensación de salud percibida por el cuidador. Del mismo modo sucede con la percepción de la fatiga, en la cual se encontró una influencia estrecha con el hecho de presentar dolores musculares.

La familia fue la principal proveedora de cuidado con $75 \%$, representando en la totalidad de familiares donde se incluye madre como principal proveedora, hijos, hermanas. En el estudio realizado por Trillos en el 2007 y otros estudios reportan que el $40 \%$ de apoyo recibido por el cuidador es por parte de hermanas y hermanos ${ }^{18,23}$. Situación que refleja la importancia de la red familiar en la provisión de cuidados.

Con respecto a las personas que reciben el cuidado, la mayoría está por encima de 65 años, y la causa principal de la discapacidad es la enfermedad general. Datos que se encuentran alrededor de los registrados con los datos nacionales ${ }^{7,8}$, donde se encuentra una mayor prevalencia de discapacidad en la población adulta, significando que la necesidad de cuidado tiende a aumentar.

En cuanto al nivel de estudios de la población en situación de discapacidad, en la mayoría se refleja el bajo o ningún nivel de escolaridad. Esto demuestra una realidad del contexto que disminuye la oportunidad de inclusión laboral y aumenta el nivel de dependencia, con posibles consecuencias para el cuidador.

Los datos encontrados en este estudio se enmarcan dentro de un grupo específico trabajado y no permite ser concluyente sobre la población cuidadora de toda la comuna 18 , de donde proviene la muestra. Sin embargo, aporta elementos que conciernen al cuidado familiar y principalmente a la persona cuidadora, de tal forma que sirvan como base para el de- sarrollo de otro tipo de estudios que profundicen sobre la experiencia del cuidado, las construcciones de red, las motivaciones e incluso la comparación entre diferentes escenarios.

De igual forma el estudio aporta insumos para que sean tenidos en cuenta en la pertinencia de las políticas públicas de acuerdo con las características del cuidado y su contexto, hace visible la población (cuidadores) que existe y requiere apoyo de las instituciones y organizaciones comunitarias, valida una metodología e instrumentos que puedan ser aplicados en otros contextos.

\section{CONCLUSIONES}

Las características y las condiciones del cuidador, representan un riesgo para la salud, lo que puede resultar determinante en la calidad del cuidado que imparte, potenciando los riesgos entre la interacción del cuidador y la persona en situación de discapacidad.

La política pública de discapacidad de Santiago de Cali de 2006, dentro de sus principios contempla la solidaridad y la corresponsabili$\mathrm{dad}^{24}$, lo cual implica la participación de diferentes actores dentro de los que se incluye la familia como generadores de solidaridad.

Teniendo en cuenta que los procesos de envejecimiento y los estados de discapacidad, conllevan a niveles de dependencia que requieren de ayuda o asistencia para algunas o todas las actividades funcionales en un individuo, situaciones que demandan de cuidado, comprometiendo a todos ${ }^{17}$; es preciso pensar desde la atención primaria en salud, las políticas públicas en el tema del cuidado ${ }^{18,}$ tema con una invisibilidad, pero con muchas relaciones sociales $^{19}$. De allí la importancia de establecer programas de atención específica y captación de problemas de salud, para evitar que este grupo ingrese a la población en situación de discapacidad.

\section{REFERENCIAS}

1. BARRERA LETAL. Cuidando a los cuidadores. Universidad Nacional de Colombia y Colciencias 2005. 
2. MURILLO DE LA VEGA S. Universidad de Salamanca Política y Sociedad, Invisibilidad del cuidado en la familia.35 (2000), Madrid. pp. 73-80.

3. ROSES MIRTA. Desigualdades Ocultas Monde Diplomatique (Número 43, enero de 2003) http://www. eldiplo.org/resumen.php3?numero $=43 \&$ resumen $=4$ 3/R 1329

4. AGÜIRRE R. Los cuidados familiares como problema público y objeto de políticas. Reunión de expertos "Políticas hacía las familias, protección e inclusión social" Cepal. Junio 2005.Pag 3.

5. GARCÍA CALVENTE M, RODRÍGUEZ I, EGUI GUREN. El sistema informal de cuidados en clave de desigualdad.. Escuela Andaluza de Salud Pública. Granada. España.2004

6. RUEDA, JO. Retos del envejecimiento demográfico en Colombia. En: Ministerio de Comunicaciones, Cepsiger. Periodismo y comunicación para todas las edades. Bogotá: El Ministerio. 2004.

7. ENCUESTA NACIONAL DE SALUD 2007.

8. SECRETARIA DE SALUD PÚBLICA MUNICIPAL 2006. Datos estadísticos.

9. DANE, Censo Nacional del 2005. Informe sobre Discapacidad

10. SALVADOR S. Estudio comparativo de economía del cuidado en Argentina, Brasil, Chile Colombia, México y Uruguay. Red internacional de género y comercio, Capítulo latinoamericano. Octubre 2007. Pag. 4.

11. GARCÍA CALVENTE M. Cuidados de salud, género y desigualdad. Escuela Andaluza de Salud Pública. Granada España 2002.

12. Documento CONPES Social 80 Consejo Nacional de Política Económica y Social. República de Colombia. Departamento Nacional de Planeación. Bogotá 2004. pág. 2.

13. WHITEHEAD M. A. Typology of actions to tackle social inequalities in health J. Epidemiol Community Health JECH 2007,61:473-478 doi:10:1136/ jech2005.037242
14. ALCALDÍA MAYOR DE BOGOTÁ. Secretaria Distrital de Planeación Encuesta de Calidad de Vida 2003. Disponible en (www.sdp.gov.co).

15. DIRECCIÓN GENERAL DE SALUD PÚBLICA. Comunidad de Madrid. Encuesta de Salud de la Comunidad de Madrid 2007.Disponible en (www.madrid.org/cs/satelite?...portalsalud).

16. TRILLOS M. Estudio de red personal en cuidadores de pacientes con alzheimer. Universidad Nacional Autónoma de México. 2007.

17. GIRALDO C., FRANCO G, CORREAL,SALAZAR M, TAMAYO A. Cuidadores familiares de ancianos quiénes son y cómo asumen ese rol. Rev. Fac Nac Salud Publica Vol. 23 N. ${ }^{\circ} 2$ jul-dic 2005. pag. 8.

18. DE LA CUESTA BENJUMEA C. Cuidado Informal en condiciones crónicas. Una aproximación a la Literatura. Texto contexto enfermería. Marzo 2004.

19. DUEÑAS E, MARTÍNEZ M, MORALES B. Síndrome del cuidador de adultos mayores discapacitados y sus implicaciones psicosociales. Colombia Medica v. 37 n.2. Junio 2006.

20. SANCHO M, ABELLAN A. RODRÍGUEZ V. La atención formal e informal en España. En Imserco, editor las personas mayores en España informe 200, documento electrónico 2000 consultado \{ Noviembre 112009$\}$ Disponible en http:/imsersomayores. csic.es

21. GIRALDO MOLINA C, FRANCO G. Calidad de vida del cuidador Revista Achichan 2002.

22. HUNG JOCIK G, SÁNCHEZ B, BELLOS M. Percepción de sobrecarga en cuidadores de pacientes con demencia. Red Latinoamericana de Gerontología. Octubre 2003.

23. DE LA CUESTA BENJUMEA C. Aquí cuidamos todos, asunto de individualidad versus colectividad. Forum: Qualitative Social Research Sozialfors Chung. Volumen 7, No. 4, Art. 5 Septiembre 2006.

24. CONCEJO DE SANTIAGO DE CALI. Documento. Acuerdo No.0197 de 2006, Política Pública de atención a la Discapacidad. Santiago de Cali.

Usted puede comentar éste y otros artículos publicados en la Revista Chilena de Salud Pública, enviando un correo electrónico a revistasp@med.uchile.cl 